\title{
A8 JKTBP (HNRNPDL) IS A NOVEL TOLL7/9 DEPENDENT TARGET IN HUMANS AND ANIMAL MODELS OF INFLAMMATORY RHEUMATIC DISEASES
}

BMarklein, ${ }^{1}$ ZKonthur, ${ }^{2} \mathrm{~A}$ Cope, ${ }^{3} \mathrm{M}$ Shlomchik, ${ }^{4} \mathrm{G}$ Burmester, ${ }^{1} \mathrm{~K}$ Skriner ${ }^{1}{ }^{1}$ Department of Rheumatology and Clinical Immunology, Humboldt University and Free University, Germany; ${ }^{2}$ Department of Vertebrate Genomics, Max-Planck Institute for Molecular Genetics, Berlin, Germany; ${ }^{3}$ Department of Rheumatology, King's College School of Medicine, London, UK; ${ }^{4}$ Yale School of Medicine, New Haven, Connecticut, USA

\subsection{6/ard.2010.129577h}

Objective Autoimmune diseases are characterised and regularly diagnosed by the presence of autoantibodies to heterogeneous nuclear RNP (hnRNP) autoantigens. These proteins represent a group of autoantigens which are targeted by autoantibodies from patients with rheumatoid arthritis (RA), systemic lupus erythematosus (SLE) and mixed connective tissue disease. The authors investigated a new member of the hnRNP family targeted by autoantibodies and tested a combination of hnRNPs in humans and animal models of inflammatory rheumatic diseases

Methods Using protein macroarray the authors identified JKDBP in humans and animal models of inflammatory rheumatic diseases. Autoantigens are produced in bacteria expressed purified using His-tag and a Biotin-tag under native and denaturated conditions. Bacterially expressed recombinant hnRNPs proteins were used in Elisa for confirming the data obtained by macroarray. Anti-hnRNP-A/B and hnRNP D proteins were detected in a newly developed Elisa with patient sera and sera from animal model of SLE and RA.

Results Autoantibodies to hnRNPDL proteins were detected in $46 \%$ of patients with SLE, $21 \%$ of patients with RA, $17 \%$ of MR1 lpr mice, $50 \%$ of SKG Mice and $<10 \%$ of patients with other rheumatic disorders. The auoantibody response is dependent on both TLR7 and TLR9 costimulation tested with sera from TLR7, TLR9 deficient and double-deficient mice with an MRL-lpr/lpr background. Using a combination of three hnRNPs A2/D/DL, $76 \%$ of RA, $84 \%$ of SLE sera and $87 \%$ SKG mice with arthritis, can be detected. Using citrullinated peptides out of a mutated form of hnRNPA3 and hnRNPA2/D/ DL, $94 \%$ out of 68 RA sera can be identified. Moreover already identified autoantigens like hnRNP A2/A3/D, identified epitopes as well as identified citrullinated peptides (deduced citrullinated peptides thereof) were used to identify patterns associated with disease severity.

Conclusion These data identify hnRNPDL proteins as novel autoantigen in RA, SLE and in animal models of inflammatory rheumatic diseases. The anti-hnRNPDL antibody is Toll 7 and 9 dependent generated and in combination with other hnRNPs D/A2/A3 they can be used to partially close the sensitivity gap left by rheumatoid factor and anti CCP antibodies in RA. 